\title{
Development of a framework for genotyping bovine-derived Cryptosporidium parvum, using a multilocus fragment typing tool
}

Emily J. Hotchkiss ${ }^{1 *}$, Janice A. Gilray ${ }^{1}$, Marnie L. Brennan², Robert M. Christley ${ }^{3}$, Liam J. Morrison ${ }^{4}$,

Nicholas N. Jonsson ${ }^{5}$, Elizabeth A. Innes ${ }^{1}$ and Frank Katzer ${ }^{1}$

\begin{abstract}
Background: There is a need for an integrated genotyping approach for C. parvum; no sufficiently discriminatory scheme to date has been fully validated or widely adopted by veterinary or public health researchers. Multilocus fragment typing (MLFT) can provide good differentiation and is relatively quick and cheap to perform. A MLFT tool was assessed in terms of its typeability, specificity, precision (repeatability and reproducibility), accuracy and ability to genotypically discriminate bovine-derived Cryptosporidium parvum.

Methods: With the aim of working towards a consensus, six markers were selected for inclusion based on their successful application in previous studies: MM5, MM18, MM19, TP14, MS1 and MS9. Alleles were assigned according to the fragment sizes of repeat regions amplified, as determined by capillary electrophoresis. In addition, a region of the GP60 gene was amplified and sequenced to determine gp60 subtype and this was added to the allelic profiles of the 6 markers to determine the multilocus genotype (MLG). The MLFT tool was applied to 140 C. parvum samples collected in two cross-sectional studies of UK calves, conducted in Cheshire in 2004 (principally dairy animals) and Aberdeenshire/Caithness in 2011 (beef animals).

Results: Typeability was $84 \%$. The primers did not amplify tested non-parvum species frequently detected in cattle. In terms of repeatability, within- and between-run fragment sizes showed little variability. Between laboratories, fragment sizes differed but allele calling was reproducible. The MLFT had good discriminatory ability (Simpson's Index of Diversity, SID, was 0.92), compared to gp60 sequencing alone (SID 0.44). Some markers were more informative than others, with MS1 and MS9 proving monoallelic in tested samples.

Conclusions: Further inter-laboratory trials are now warranted with the inclusion of human-derived C. parvum samples, allowing progress towards an integrated, standardised typing scheme to enable source attribution and to determine the role of livestock in future outbreaks of human C. parvum.
\end{abstract}

Keywords: Cryptosporidium parvum, Multilocus, Genotyping, Bovine

\section{Background}

The protozoan parasite Cryptosporidium is an enteropathogen of the Apicomplexa phylum, with 26 defined species and more than 60 genotypes which have not been assigned species status [1]. Some of these species/ genotypes are strongly host associated $(C$. hominis, $C$. bovis) whereas others are capable of infecting and causing

\footnotetext{
*Correspondence: emily.hotchkiss@moredun.ac.uk

${ }^{1}$ Moredun Research Institute, Pentlands Science Park, Bush Loan, Penicuik, Edinburgh EH26 OPZ, UK

Full list of author information is available at the end of the article
}

disease in a wide range of host species. C. parvum is prevalent worldwide and infects both humans and animals, with serious clinical outcome in some populations. Neonatal livestock have been shown to shed this species in large numbers $[2,3]$ and are thought to be the most important zoonotic source of infection for humans; in the UK, it is estimated that almost all dairy and beef farms are infected and longitudinal studies suggest that approximately $100 \%$ of all calves will shed the oocysts at some point in the first few weeks of life (Thomson, unpublished data). Zoonotic and anthroponotic transmission can occur directly, via 
contact with shedding individuals, or indirectly, with the highly resistant oocysts being ingested via drinking or recreational water or food.

The different species of Cryptosporidium cannot be distinguished by microscopy therefore molecular tools are invaluable in assigning species and determining zoonotic potential. Many laboratories subtype within C. parvum and C. hominis by sequence analysis of the polyserine tract in the GP60 gene, which has variable numbers of TCA and TCG codons between subtypes [4]. Global epidemiological studies have utilised this typing tool to identify host substructuring within C. parvum, as allele IIc [5], for example, has only been found to date in humans.

At a national or regional level, it is important to determine subtypes of a species to aid in source attribution in the event of human infection. In this case, sequencing of the GP60 gene alone is unlikely to provide sufficient levels of discrimination, particularly where common subtypes exist and circulate widely within potential source populations, for example gp60 subtype IIaA15G2R1, which is prevalent worldwide in both humans and cattle $[6,7]$. To enable rapid investigation in the event of an outbreak of cryptosporidiosis in humans, harmonised methods of molecular typing must be adopted by both public health agencies and workers in the veterinary field. Knowledge of genotypes circulating in potential source populations, such as livestock and wildlife, would be invaluable. However, whether these baseline data are available or not, the ability to easily, robustly and rapidly screen potential sources in real time is an essential requirement of any tool to be used within an outbreak situation.

With time, the field of genomics may provide the gold standard in discriminatory typing. However genomics within the field of Cryptosporidium has been lagging behind other genera, possibly due to the lack of an in vitro culture system and issues with the low GC composition and poly T/A runs; only 3 published genomes are currently available. Much of the work to date at the genome level has focused on differences between Cryptosporidium species, with a current paucity of data on within-species comparisons.

Multilocus fragment typing (MLFT) uses tandem repeat units within the genome, often called micro- or mini-satellites, within coding or non-coding regions. Mini/micro-satellite regions have been shown to provide good differentiation in moderately conserved species. Multiple regions, or markers, are amplified by PCR and the resulting amplicons are sized by gel or capillary electrophoresis (CE). Length polymorphisms due to variable numbers of nucleotide repeats are the basis for genotyping and the alleles at the different markers are combined to give a multilocus genotype (MLG). Using CE, one primer is given a fluorescent label to allow sizing. Fragment sizing is preferable to sequence analysis as this can often be challenging across repeat regions. Another advantage of MLFT over multilocus sequence typing (MLST), as trialled primarily with $C$. hominis [8-10], is that samples containing mixed genotypes are readily identified by secondary peaks on trace files. Studies have demonstrated that mixed genotypes occur in cattle faeces at variable rates from 0.8 to $37 \%$ depending on the geographic region $[11,12]$. In addition, fragment sizing is rapid and costeffective, which may be important in an outbreak situation. Whereas the use of MLFT within a laboratory may be informative, technical difficulties arise when comparing results between laboratories. It has been shown that the observed sizes depend on more than the actual length of the fragments amplified; other potential factors include sequence composition and DNA conformation, the machine used and running conditions [13, 14].

A recent review of the use of MLFT in published Cryptosporidium research has highlighted the lack of an integrated approach or validation of this, potentially valuable, typing tool [15]. In the current study we used markers which appeared to be informative from previous studies in order to move towards a consensus for C. parvum. We applied this tool to bovine C. parvum samples and report on the performance of the tool in terms of sensitivity (typeability), specificity, discriminatory ability, repeatability and reproducibility.

\section{Methods}

\section{Study population}

Faecal samples from two cross-sectional studies of UK calves were processed by standard methods [7, 16]. Briefly, approximately $200 \mu \mathrm{g}$ of faeces was placed in lysis buffer and subjected to freeze-thaw cycles before being processed by QIAamp DNA Stool Minikit (Qiagen). Those confirmed as C. parvum $(n=140)$ by sequence analysis of the $18 \mathrm{~S}$ rRNA locus [17] were further characterised by MLFT. The C. parvum samples were sourced from: a) 92 calves from 21 beef farms in Aberdeenshire and Caithness, Northeast Scotland, collected in Spring 2011, and b) 48 calves from 21 (predominantly dairy) farms, Cheshire, Northwest England, collected in Spring $2004[7,16]$.

\section{Markers}

A panel of 6 markers was selected based on previous studies: MM5, MM18, MM19, TP14, MS1 and MS9 (Table 1). In addition, a region of the GP60 gene was amplified as previously described [7] and sequenced to provide gp60 subtype, which was added to the allelic profile to assign multilocus genotypes (MLGs). External primers were designed to those in the literature for use in the first round of a nested PCR reaction (Table 2) 
Table 1 Markers selected for multilocus fragment typing of cattle-derived Cryptosporidium parvum

\begin{tabular}{llll}
\hline Marker & Chromosome & Repeat & Reference \\
\hline MM5 & 6 & TC(T/C) & {$[12]$} \\
MM18 & 8 & (C/G)CAG(A/G)A & {$[12]$} \\
MM19 & 8 & GGAGCT & {$[12]$} \\
TP14 & 8 & CAA & {$[20]$} \\
MS1 & 2 & GG(C/T)GG(T/A)ATGCCA & {$[33]$} \\
MS9 & 5 & TGGATC & {$[19]$} \\
gp60 & 6 & TC(A/G) & {$[4]$} \\
\hline
\end{tabular}

${ }^{\mathrm{a}}$ gp60 was subjected to sequence analysis

[18]. FAM or HEX was used to 5' label one of the second round primers (MWG Eurofins).

\section{PCR}

Nested PCR was carried out. Mastermix consisted of 10X PCR buffer which was prepared in house using $45 \mathrm{mM}$ Tris-HCl (Trizma base, Sigma; $\mathrm{HCl}$ : Fisher Scientific), $11 \mathrm{mM} \quad\left(\mathrm{NH}_{4}\right)_{2} \mathrm{SO}_{4}, 4.5 \mathrm{mM} \quad \mathrm{MgCl}_{2}$, $0.113 \mathrm{mg} / \mathrm{ml} \mathrm{BSA}, 4.4 \mu \mathrm{M}$ EDTA (all Sigma) and $1.0 \mathrm{mM}$ dATP, dATC, dGTP, dTTP (VH Bio Ltd). One unit of Biotaq (Bioline), 20 pmol primer and 1ul DNA template was added in a 20 ul reaction volume. PCR reactions were performed in a G-Storm thermocycler (Gene Technologies Ltd) and cycling conditions were 30 cycles of $95^{\circ} \mathrm{C}$ for $50 \mathrm{~s}, 50{ }^{\circ} \mathrm{C}$ for $50 \mathrm{~s}$ and $65^{\circ} \mathrm{C}$ for $60 \mathrm{~s}$. PCR product from the primary reaction was diluted 1:100 before use as template in the second round PCR.

\section{Fragment analysis}

Labelled PCR products were subjected to fragment analysis using capillary electrophoresis (CE) via ABI 3730
(Applied Biosystems; University of Dundee), using size standard Genescan ROX500 (Applied Biosystems). A subset of samples was also re-sized using ROX400HD (Applied Biosystems). Trace files were then analysed using both Peak Scanner (Applied Biosystems) and STRand (https://www.vgl.ucdavis.edu/informatics/strand.php). Secondary peaks, representing mixed genotype infections, were assigned when their height was $\geq 0.33$ of the primary peak and where the size of the secondary fragment had been detected in other isolates as a primary peak.

\section{Typeability and specificity}

Typeability was assessed for each marker as well as for the combined MLFT/gp60 tool; it was calculated as the number of samples which were successfully assigned an allele number or MLG divided by the total number of samples trialled. PCRs were repeated for samples which did not produce an amplicon at one or more markers.

In order to verify specificity of the primers (i.e., their ability to exclusively amplify the target DNA), a selection of non-C. parvum species likely to be present in cattle samples were included: $C$. bovis $(n=3), C$. ryanae $(n=1)$ and $C$. andersoni $(n=1)$. Samples were single-species infections as determined by multiplex PCR (data not shown) and confirmed by sequence analysis of the $18 \mathrm{~S}$ rRNA gene. These samples were processed with MLFT primers in triplicate PCR reactions. In addition, specificity was tested using primer-BLAST to ascertain whether the primers aligned with sequences deposited in GenBank and were therefore likely to amplify other organisms or non-parvum Cryptosporidium species.

Table 2 Primers used in nested PCR reactions in multilocus fragment typing of cattle-derived Cryptosporidium parvum

\begin{tabular}{|c|c|c|c|c|}
\hline Marker & & Forward & Reverse & Reference \\
\hline \multirow[t]{2}{*}{ MM5 } & $1^{\circ}$ & TCACAAGTTACCCCTTCTGATGCTG & TCCACCTCCGGATTGGTTGTG & [18] \\
\hline & $2^{\circ}$ & CCTGGACTTGGATTTGGACTTACACC & GGAGAAGATAAGCTAGCCGAATCT & {$[12]$} \\
\hline \multirow[t]{2}{*}{ MM18 } & $1^{\circ}$ & GTTCAGCTGATACGGGTTTGCAACA & CATCACCATCTCCTCCGCCAGA & {$[18]$} \\
\hline & $2^{\circ}$ & CTITCTGGAGGGTTTGTTCCTCC & СTTCCTGATGATCCAGGCCAAGC & [12] \\
\hline \multirow[t]{2}{*}{ MM19 } & $1^{\circ}$ & TGGTIITAGCTAAGGAAGCGATAG & CTGCTGCTGCTGTTGCTITA & [18] \\
\hline & $2^{\circ}$ & GATTCTGTCAACTTTGAATTCAG & CCAACCCCGAATTCATTTCCAAC & {$[12]$} \\
\hline \multirow[t]{2}{*}{ TP14 } & $1^{\circ}$ & GAGAAGGAGCAATGGGAGCA & TCCTCCTITTTGCCCTTGAA & [18] \\
\hline & $2^{\circ}$ & CTAACGTTCACAGCCAACAGTACC & CAATAAAGACCATTATTACCACC & [20] \\
\hline \multirow[t]{2}{*}{ MS1 } & $1^{\circ}$ & AAGGGTGAGGATGAGCAGAA & TTCTTAACTTTCCATTTTGAGTGA & Current study \\
\hline & $2^{\circ}$ & TTAGTCGACCTCTTCAACAGTTGG & GGAACACCATCCAAGAACCAAAGGT & [26] \\
\hline \multirow[t]{2}{*}{ MS9 } & $1^{\circ}$ & TTAGTCGACCTCTTCAACAGTTGG & CAGAAT TGGAATCATITTCTGAAT & Current study \\
\hline & $2^{\circ}$ & GGACTAGAAATAGAGCTITGGCTGG & GTCTGAGACAGAATCTAGGATCTAC & [19] \\
\hline \multirow[t]{2}{*}{ gp60 } & $1^{\circ}$ & ATAGTCTCCGCTGTATTC & GAGATATATCTTGGTGCG & [7] \\
\hline & $2^{\circ}$ & TCCGCTGTATTCTCAGCC & CGAACCACATTACAAATGAAG & [7] \\
\hline
\end{tabular}




\section{Precision: repeatability and reproducibility}

In order to determine repeatability of fragment sizing, a subset of the samples was amplified and sized on several different occasions and fragment sizes compared between runs (between-run variation). In addition, the same sample was repeated within a PCR plate and sized within a run (within-run variation). A representative of each known allele was included in every PCR run and sizing plate.

To assess reproducibility, bovine-derived C. parvum DNA was made available which had previously been amplified and sized in another laboratory [12, 19, 20]. These samples were processed in our laboratory to assess the effect of machine and running conditions on the reproducibility of both fragment sizing and allele assignation.

\section{Discriminatory ability}

Simpson's Index of Diversity (SID) was used to assess discriminatory ability. This value provides an estimate of the probability that two epidemiologically-unrelated samples selected at random will be different alleles/ genotypes [21]. SID with $95 \%$ confidence interval was calculated using V-DICE (Variable Number Tandem Repeat Diversity and Confidence Extractor; http:// www.hpa-bioinformatics.org.uk/cgi-bin/DICI/DICI.pl) for differentiation of C. parvum into a) different alleles for each marker, b) MLGs using MLFT and c) gp60 subtypes using sequence analysis of gp60 alone. Where mixed profiles were obtained, only the primary peak was used to assign alleles for this analysis.

\section{Results}

\section{Typeability and specificity}

Within the Scottish survey, 92 samples were C. parvumpositive and of these, 2 samples failed to amplify with gp60 primers and one amplicon gave a mixed gp60 profile. Within Cheshire samples 1/48 samples gave a mixed gp60 profile and therefore we were unable to conclusively assign a gp60 subtype. Overall therefore the typeability of gp60 sequencing was $136 / 140$ or 0.97 , with $95 \%$ confidence interval (CI) of 0.93-0.99 (Table 3).

All 92 Scottish C. parvum samples were subjected to MLFT at 6 markers. Of these, 82 were successfully assigned an allele at all 6 markers. Within Cheshire samples, there was insufficient DNA template resulting in some missing data for markers MS1 $(n=5)$ and MS9 $(n=26)$. Typeability for these markers was calculated using adjusted denominators (Table 3). 38/48 Cheshire samples were successfully assigned an allele at the 4 remaining markers. Samples occasionally failed to amplify at one or more loci, despite repeated attempts. Of these, 7/20 failed to amplify at any of the tested loci.

Overall, excluding MS1 and MS9 due to missing data, $118 / 140$ samples were typed with the combined scheme of 4 MLFT markers and gp60 sequencing, resulting in typeability of 0.84 (95\% CI $0.77-0.90$ ) (Table 3). No marker was significantly different in terms of typeability, ranging from 0.90 (TP14) to 0.96 (MS9) (Table 3).

None of the non-C. parvum samples amplified with any of the primers. Primer-BLAST results indicated that $C$. hominis would be co-amplified by these primers, with $100 \%$ match for MM18 primers with $C$. hominis accession number XM_661101, 1 base difference in MM5_reverse and MS1_reverse when aligned with $C$. hominis sequences (XM_661101 and XM_661662) and 4 bases differing in MS9_forward and C. hominis XM_660477. For MM19, both the $C$. parvum and $C$. hominis reference genomes (XM_627368 and XM_660787) were missing base 8 of our forward primer sequence, but the reverse primer aligned with both species $100 \%$. No other Cryptosporidium spp. aligned with these primers.

\section{Precision: repeatability and reproducibility}

In terms of repeatability, within-run variation was assessed as the range of fragment sizes obtained when the same DNA sample was amplified several times within a PCR reaction. In all cases variation was negligible $(<1 \mathrm{bp})$. Between-run variation was assessed as the range in sizes obtained when the same DNA was amplified in different PCR reactions. Across all markers the median range of sizes produced was minimal at 0.4 bp (Table 4), although MM5 allele 2 had a lower repeatability with a range of $1.9 \mathrm{bp}$ in seven replicates. Allele calling was straightforward however by comparing with sizes of the known alleles which were included in each run as controls.

For reproducibility, sizes were shown to differ between laboratories, generally within the range of 1-2 bp (Table 5). The major difference was in marker MS9 where all alleles differed in measured size by a consistent $11 \mathrm{bp}$; this difference was maintained after repeating the amplification and sizing. Allele assignment was concordant.

\section{Accuracy}

Representatives of all identified alleles were subjected to sequence analysis. All sequences analysed showed that the different alleles varied only in the repeat regions (Fig. 1) and that the difference in fragment size corresponded to variable numbers of repeat motifs. As well as aligning with the $C$. parvum reference genome, some of the alleles matched "microsatellite" sequences already deposited in GenBank from similar studies of C. parvum $[22,23]$ with $0-2$ base differences: MM5 allele 2 (JX413509), MM18 allele 2 (JX413498), MM19 allele 1 (JX413503), MM19 allele 3 (JX413502), TP14 allele 1 (JF342561) and TP14 allele 2 (JF342562). Where present, base differences were outwith the repeat region and for TP14 and MM19 were within a primer binding site. 
Table 3 Multilocus fragment typing of Cryptosporidium parvum samples sourced from 118 UK calves

\begin{tabular}{|c|c|c|c|c|c|c|c|c|}
\hline & MM5 & MM18 & MM19 & TP14 & MS1 & MS9 & gp60 & MLG \\
\hline & 235 & 288 & 298 & 296 & 361 & 455 & IlaA15G2R1 & 1 \\
\hline & 262 & 288 & 298 & 304 & 361 & 455 & IlaA15G2R1 & 2 \\
\hline & 235 & 288 & 298 & 296 & 361 & 455 & IlaA17G1R1 & 3 \\
\hline & 262 & 288 & 304 & 296 & 361 & 455 & IlaA15G2R1 & 4 \\
\hline & 262 & 294 & 292 & 304 & 361 & 455 & IlaA15G2R1 & 5 \\
\hline & 262 & 288 & 292 & 296 & 361 & 455 & IlaA15G2R1 & 6 \\
\hline & 262 & 294 & 292 & 304 & 361 & 455 & IlaA17G1R1 & 7 \\
\hline & 235 & 288 & 292 & 296 & 361 & 455 & IlaA18G3R1 & 8 \\
\hline & 262 & 294 & 298 & 296 & 361 & 455 & IlaA17G1R1 & 9 \\
\hline & 262 & 294 & 298 & 304 & 361 & 455 & IlaA15G2R1 & 10 \\
\hline & 262 & 288 & 292 & 304 & 361 & 455 & IlaA15G2R1 & 11 \\
\hline & 262 & 288 & 298 & 296 & 361 & 455 & IlaA15G2R1 & 12 \\
\hline & 235 & 288 & 270 & 296 & 361 & 455 & IlaA15G2R1 & 13 \\
\hline & 262 & 288 & 316 & 296 & 361 & 455 & IlaA15G2R1 & 14 \\
\hline & 262 & 288 & 292 & 296 & 361 & 455 & IlaA17G1R1 & 15 \\
\hline & 262 & 288 & 253 & 296 & 361 & 455 & IlaA15G2R1 & 16 \\
\hline & 262 & 288 & 298 & 296 & 361 & 455 & IlaA16G3R1 & 17 \\
\hline & 262 & 288 & 298 & 296 & 361 & 455 & IlaA19G1R1 & 18 \\
\hline & 262 & 318 & 298 & 296 & 361 & 455 & IlaA15G2R1 & 19 \\
\hline & 262 & 288 & 298 & 296 & 361 & 455 & IlaA17G1R1 & 20 \\
\hline & 262 & 294 & 281 & 296 & 361 & 455 & IlaA15G2R1 & 21 \\
\hline & 262 & 294 & 298 & 296 & 361 & 455 & IlaA15G2R1 & 22 \\
\hline & 262 & 294 & 292 & 296 & 361 & 455 & IlaA18G1R1 & 23 \\
\hline Typeability & 0.91 & 0.92 & 0.91 & 0.90 & 0.93 & 0.96 & 0.97 & 0.84 \\
\hline$(95 \%$ Cl) & $(0.85-0.95)$ & $(0.86-0.96)$ & $(0.86-0.95)$ & $(0.84-0.94)$ & $(0.87-0.96)$ & $(0.90-0.99)$ & $(0.93-0.99)$ & $(0.77-0.90)$ \\
\hline SID $(95 \%$ Cl) & $0.35(0.26-0.43)$ & $0.41(0.32-0.50)$ & $0.60(0.54-0.67)$ & $0.35(0.26-0.43)$ & $0.00(0.00-0.06)$ & $0.00(0.00-0.06)$ & $0.44(0.33-0.54)$ & $0.92(0.90-0.94)$ \\
\hline
\end{tabular}

gp60 subgenotypes are included in multilocus genotype (MLG) assignment. Typeability and Simpson's Index of Diversity (SID) are reported for allele and MLG assignment, with $95 \%$ confidence intervals $(95 \% \mathrm{Cl})$

Sequences of alleles have been deposited in GenBank under accession numbers KP172504-KP172519 (Table 6).

The size of sequenced products differed from those determined by fragment analysis (Table 6); sizes obtained by $C E$ were larger than sizes obtained by sequencing for markers MM5, MM19 and MS9 but sequenced sizes were larger for markers MM18, TP14 and MS1.

\section{Discriminatory ability}

Out of the 6 markers trialled with fragment typing, 2 were found to be monoallelic (MS1 and MS9), and two were biallelic (MM5 and TP14) (Table 3). MM18 had 3 alleles but MM19 was the most polymorphic marker, with 7 alleles identified. MM19 was confirmed as the most discriminatory marker (SID 0.60 ) followed by $g p 60$ then MM18 (Table 3).

There was limited evidence of mixed genotype infections within individual calves, with 109/118 calves shedding single alleles at each locus. In addition single MLGs were detected on most farms. Overall 23 MLGs were identified in 118 samples, from 39 different farms in two regions of the UK (Fig. 2). The majority of MLGs (14/23) were only identified on one sampled farm, however some MLGs were more widely distributed with 4 MLGs common to both the Cheshire (2004) and NE Scotland (2011) studies. One MLG was particularly prevalent, having been identified on 8 farms representing both studies (MLG 12).

SID for the MLFT tool was $0.92(0.90-0.94)$, compared to $0.44(0.33-0.54)$ by $g p 60$ sequence typing alone. Using the three most informative markers (MM19, gp60 and MM18) would have differentiated 18/23 MLGs with an overall SID of 0.85 (0.81-0.90). The four most informative markers (MM19, gp60, MM18 and TP14) would have differentiated 21/23 MLGs with a SID of 0.89 (0.85-0.92).

\section{Discussion}

As with diagnostic test development, validation of any microbial typing system is essential. Guidelines have 
Table 4 Mean fragment sizes of Cryptosporidium parvum DNA samples amplified in multiple separate PCR reactions

\begin{tabular}{llcccc}
\hline Marker & Allele & Mean Size (bp) & SE Mean (bp) & Range (bp) & N \\
\hline MM5 & 1 & 262.5 & 0.08 & 0.6 & 7 \\
& 2 & 235.3 & 0.25 & 1.9 & 7 \\
MM18 & 1 & 288.3 & 0.04 & 0.4 & 9 \\
& 2 & 293.9 & 0.05 & 0.3 & 8 \\
& 3 & 318.1 & 0.05 & 0.3 & 5 \\
MM19 & 1 & 297.7 & 0.04 & 0.3 & 8 \\
& 2 & 303.8 & 0.04 & 0.3 & 9 \\
& 3 & 292.1 & 0.06 & 0.5 & 8 \\
& 4 & 316.4 & 0.09 & 0.3 & 3 \\
& 5 & 269.8 & 0.11 & 0.6 & 5 \\
& 6 & 252.8 & 0.10 & 0.6 & 6 \\
& 7 & 281.1 & 0.07 & 0.4 & 5 \\
TP14 & 1 & 295.9 & 0.12 & 0.7 & 6 \\
& 2 & 304.7 & 0.14 & 0.8 & 6 \\
MS1 & 1 & 361.2 & 0.06 & 0.4 & 6 \\
MS9 & 1 & 455.1 & 0.04 & 0.3 & 6 \\
\hline
\end{tabular}

$N$ number of replicates. Median range $=0.4 \mathrm{bp}$

been set out for bacterial typing systems [24, 25], and many of these guidelines can be applied to parasites, particularly haploid protozoa such as Cryptosporidium. In the current study, performance of MLFT was assessed in bovine-derived C. parvum and found to provide good typeability, specificity, precision and discrimination.

Markers were selected as they had shown promising results in other studies of bovine C. parvum [12, 22, 26], and were also ranked highly in a recent review of published multilocus genotyping methods [15]. An ideal typing scheme would have markers distributed evenly across several chromosomes [27], which is not the case in the current scheme - of the 4 most discriminatory markers, three are on Chromosome 8. MM18 and MM19 are some distance from each other, although TP14 and MM18 are somewhat closer on Chromosome 8. Selecting markers on different chromosomes would remove any confounding effect of physical linkage and provide the added value of enabling data generated to be analysed robustly at the population genetic level. As more genomic data becomes available for $C$. parvum, it should be possible to select more appropriate markers for MLFT schemes.

Typeability using nested PCR was considered acceptable at $84 \%$. It was observed that samples which were variably amplified with $18 \mathrm{~S}$ rRNA primers (for example only amplifying in one or two of three replicates) were also difficult to amplify using MLFT primers (data not shown) and 7 samples failed to amplify with any of the MLFT primers. This suggests that these samples contained low levels of, or poor quality, C. parvum DNA, as the $18 \mathrm{~S}$ rRNA protocol has been shown to be very sensitive, perhaps due to the multi-copy nature of this gene. DNA was prepared from stool using standard methods, however template quality may have adversely affected typeability. The DNA samples from the Cheshire study were prepared in 2004, 10 years before use in the current study, possibly allowing degradation of DNA. In addition, samples may have contained low numbers of oocysts as calves were not sampled on the basis of clinical signs in either study - many calves were not in the acute stage of infection. Typeability obtained with MLFT has been shown to compare favourably with MLST [13], possibly due to the "stutter effect" where tandem repeat units interfere with sequencing.

Although $C$. parvum is the most prevalent species in young calves, $C$. bovis and C. ryanae are also occasionally identified, whereas $C$. andersoni is usually found in older animals [7, 28]. We wanted to verify that the primers used would not co-amplify any non-C. parvum species in undetected mixed species infections, as this could be misinterpreted as a new (C. parvum) allele. The current study trialled a limited number of non-parvum species prevalent in bovines and found that the primers did not amplify these species. However, where environmental, human or wildlife samples are to be typed a greater range of species may be identified, therefore primer-BLAST was used and it was established that $C$. hominis could be co-amplified with these primer pairs, as shown in previous studies using these markers [26]. This may be of value to public health laboratories as the same scheme could potentially be used for both of the major causes of cryptosporidiosis in humans. However a comprehensive review of MLFT markers in both species concluded that different sets of markers are probably required for each species [15]. It is always advisable to first assign samples to species level before further typing.

Precision, in terms of the repeatability of sizes obtained within our laboratory, was good, with the possible exception of MM5 allele 2. Between laboratories, fragment sizes did differ to some degree resulting in reduced reproducibility of sizing. The consistent difference of $11 \mathrm{bp}$ in MS9 sizing remains to be explained and unfortunately sequencing data is not available for the historical data. We consider it unlikely that this is a true reproducibility issue, given that it is limited to this marker however MS9 was excluded from further analysis as it was monoallelic in our samples, along with marker MS1. Therefore there are challenges in comparing MLFT results between laboratories unlike, for example, sequence data; however, crucially, our results show that the tool is reproducible with respect to allele assignation. Larger scale inter-laboratory validation is now warranted. In the future it may be beneficial to have marker-specific size standards which include sizes of all known alleles, aiding reproducibility. 
Table 5 Comparison of fragment sizes obtained when bovine Cryptosporidium parvum DNA was processed in two different laboratories

\begin{tabular}{|c|c|c|c|c|c|}
\hline \multirow[t]{2}{*}{ Marker } & \multirow{2}{*}{$\begin{array}{l}\text { MRI } \\
\text { allele }\end{array}$} & \multicolumn{2}{|l|}{ MRI } & \multicolumn{2}{|l|}{ UoG } \\
\hline & & Size (bp) & Prevalence $(n=118)$ & $\overline{\text { Size }(b p)^{a}}$ & Prevalence $(n=211)$ \\
\hline \multirow[t]{3}{*}{ MM5 } & 1 & 262 & $78 \%$ & 260 & $69 \%$ \\
\hline & 2 & 235 & $22 \%$ & 233 & $29 \%$ \\
\hline & NA & 288 & $0 \%$ & 287 & $0.5 \%$ \\
\hline \multirow[t]{4}{*}{ MM18 } & 1 & 288 & $73 \%$ & 290 & $56 \%$ \\
\hline & 2 & 294 & $22 \%$ & 296 & $2 \%$ \\
\hline & 3 & 318 & $4 \%$ & NA & $0 \%$ \\
\hline & NA & 299 & $0 \%$ & 302 & $0.5 \%$ \\
\hline \multirow[t]{8}{*}{ MM19 } & 1 & 298 & $53 \%$ & 299 & $38 \%$ \\
\hline & 2 & 304 & $4 \%$ & 305 & $4 \%$ \\
\hline & 3 & 292 & $33 \%$ & 293 & $55 \%$ \\
\hline & 4 & 316 & $2 \%$ & NA & $0 \%$ \\
\hline & 5 & 270 & $2 \%$ & 269 & $0.5 \%$ \\
\hline & 6 & 253 & $3 \%$ & NA & $0 \%$ \\
\hline & 7 & 281 & $3 \%$ & 281 & $0.5 \%$ \\
\hline & NA & 310 & $0 \%$ & 311 & $1 \%$ \\
\hline \multirow[t]{2}{*}{ TP14 } & 1 & 296 & $78 \%$ & 297 & $60 \%$ \\
\hline & 2 & 305 & $22 \%$ & 306 & $40 \%$ \\
\hline \multirow[t]{3}{*}{ MS1 } & 1 & 361 & $100 \%$ & 362 & $99 \%$ \\
\hline & NA & 327 & $0 \%$ & 326 & $0.5 \%$ \\
\hline & NA & 384 & $0 \%$ & 386 & $0.5 \%$ \\
\hline \multirow[t]{3}{*}{ MS9 } & 1 & 455 & $100 \%$ & 444 & $96 \%$ \\
\hline & NA & 443 & $0 \%$ & 432 & $1.5 \%$ \\
\hline & NA & 461 & $0 \%$ & 450 & $2 \%$ \\
\hline
\end{tabular}

DNA was prepared and initially sized at the University of Glasgow (UoG), then amplified and sized at Moredun Research Institute (MRI). In addition the prevalence of each allele is given for the two studies. Only primary peaks were used to assign allele number

${ }^{a}$ Allele sizes are the binned Genescan results

The current protocol may not accurately measure size, as demonstrated when sequence and fragment sizes were compared. Again, measuring the size accurately may not be as important as assigning the correct alleles. Sequence and fragment size obtained by $\mathrm{CE}$ have been shown to differ in other studies [13]. One aspect that may affect accuracy is the size standard used. ROX400HD has 21 size markers, compared to 16 for ROX500, therefore it is more accurate in sizing fragments up to $400 \mathrm{bp}$. In addition, Applied Biosystems' literature states that the marker at 250 bp cannot be used to size samples with ROX500 as it is sensitive to small temperature variations in CE. Fragments in this range may be sized less accurately, which may particularly impact on MM5, with alleles of 235 bp and $262 \mathrm{bp}$. However, MS9 has fragments $>400 \mathrm{bp}$ so ROX400HD could not be used for this marker; for consistency, ROX500 was used throughout.

The level of discrimination required by a particular typing tool depends on the epidemiological question being addressed. The population genetics of the microbe should also be considered. Here we were seeking a tool to answer geographically and temporally local epidemiological questions in a relatively conserved parasite. In Cryptosporidium, the ideal MLFT tool should have the discriminatory ability to differentiate geographically local isolates [27], and for this reason we used isolates from two cross-sectional studies, which sampled farms from relatively small spatio-temporal windows. The results show that the typing scheme was able to fulfil this criterion, as $14 / 23$ MLGs were unique to sampled farm although most of the MLGs detected were part of the same clonal complex (data not shown). In addition, the finding that most calves and farms had single MLGs suggests that the scheme is not overly discriminatory for regional (such as catchment-level) studies. An application of this tool is to study transmission dynamics between and within farms, by investigating whether farms have "unique" or "common" MLGs, single or multiple MLGs and to investigate stability over time (manuscript in preparation). 


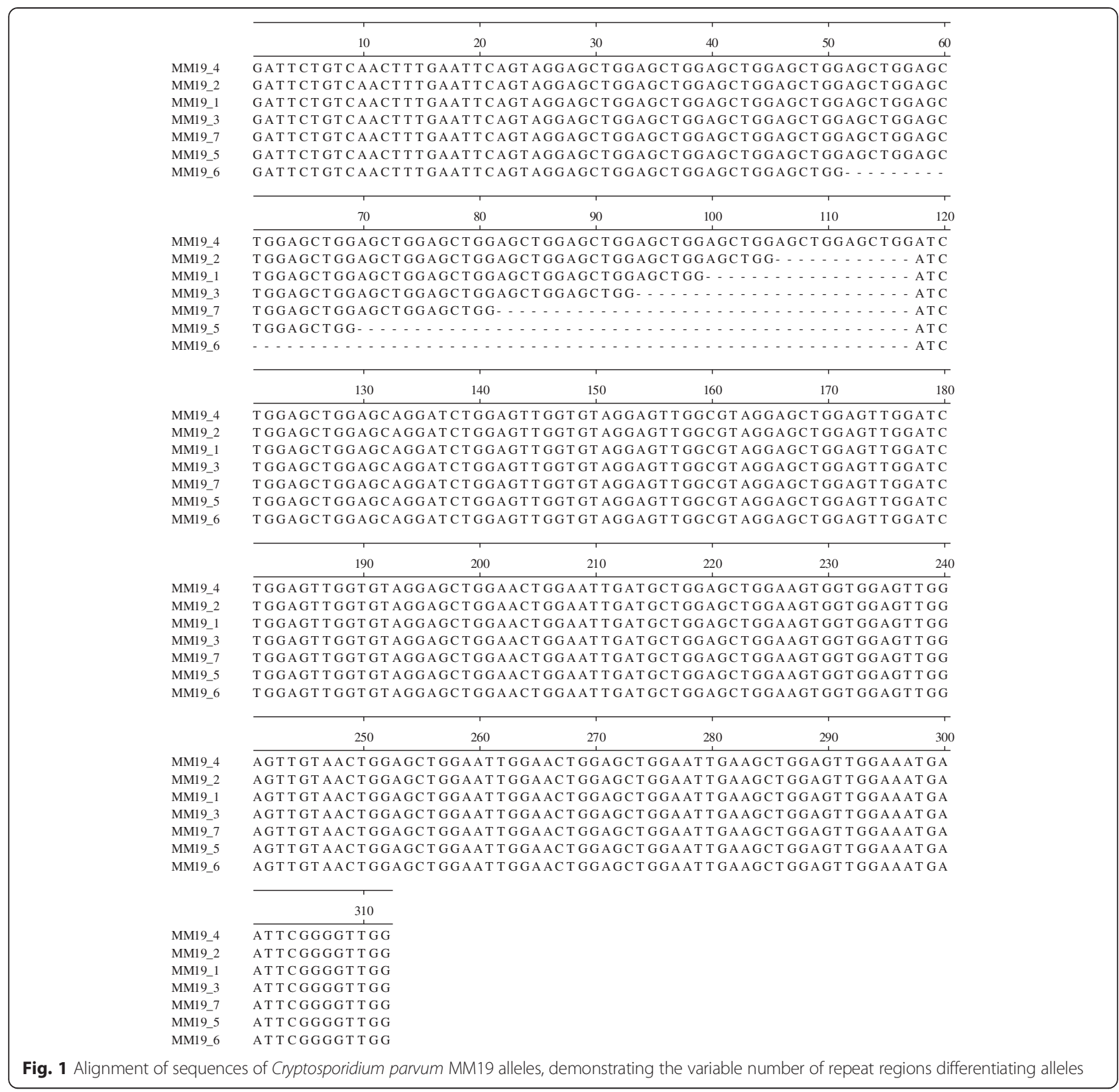

The MLFT scheme showed good discriminatory power when compared to standard subtyping using gp60 sequencing alone, as demonstrated by SID. This is due to the fact that the majority $(85 / 136)$ of samples were the common gp60 subtype, IIaA15G2R1. As samples were not independent but were clustered by farm, the values for SID may not be applicable to the general cattle population - in fact, the non-independence may actually reduce the apparent SID. Some markers were more informative than others - MS1 and MS9 were monoallelic in the samples tested. However, some diversity has been reported with these markers in other studies of cattle $[11,12]$, although the prevalence of alleles other than those in the current study appear to be very rare in Scottish and Irish calves [12, 22]. Widmer and Sullivan [27] recommended that the minimum number of markers be used to give the required resolution; a recent review estimated that in C. parvum there was, on average, $23 \%$ marker redundancy [15]. This is true of the current study, demonstrated by the fact that SID using just the three or four most informative markers was estimated at $85 \%$ (81-90\%) and $89 \%$ (85-92\%) respectively.

Other studies utilising fragment sizing have included a similar region of the GP60 gene to that used in sequence analysis for subtype assignment; when used in this way it is often referred to as GP15. We chose to use sequence 
Table 6 Sizes of fragments of Cryptosporidium parvum DNA obtained using capillary electrophoresis compared with sizes obtained by sequence analysis

\begin{tabular}{|c|c|c|c|c|}
\hline \multirow[t]{2}{*}{ Marker } & \multirow[t]{2}{*}{ Allele } & \multicolumn{2}{|l|}{ Size (bp) } & \multirow{2}{*}{$\begin{array}{l}\text { Accession } \\
\text { number }\end{array}$} \\
\hline & & Fragment analysis & Sequence analysis & \\
\hline \multirow[t]{2}{*}{ MM5 } & 1 & 261.4 & 260 & KP172504 \\
\hline & 2 & 234.6 & 233 & KP172505 \\
\hline \multirow[t]{3}{*}{ MM18 } & 1 & 288.1 & 290 & KP172506 \\
\hline & 2 & 293.7 & 296 & KP172507 \\
\hline & 3 & 317.5 & 320 & KP172508 \\
\hline \multirow[t]{7}{*}{ MM19 } & 1 & 297.6 & 294 & KP172509 \\
\hline & 2 & 303.7 & 300 & KP172510 \\
\hline & 3 & 292.0 & 288 & KP172511 \\
\hline & 4 & 316.3 & 312 & KP172512 \\
\hline & 5 & 269.1 & 264 & KP172513 \\
\hline & 6 & 252.0 & 246 & KP172514 \\
\hline & 7 & 280.3 & 276 & KP172515 \\
\hline \multirow[t]{2}{*}{ TP14 } & 1 & 295.6 & 301 & KP172516 \\
\hline & 2 & 304.3 & 310 & KP172517 \\
\hline MS1 & 1 & 361.2 & 362 & KP172518 \\
\hline MS9 & 1 & 455.1 & 450 & KP172519 \\
\hline
\end{tabular}

analysis for this gene as this method is a good library typing tool, having been adopted almost universally by Cryptosporidium researchers worldwide allowing for easy comparison of subtypes. Being sequence based it offers more discrimination than fragment sizing alone but mixed profiles can be problematic. As shown in the current study, the discriminatory ability of this single locus sequence type is not sufficient for local epidemiological questions, such as outbreak investigations.

Where harmonised schemes are being developed to allow source attribution, it should be considered whether markers are informative in C. parvum derived from both potential sources of oocysts (livestock, wildlife

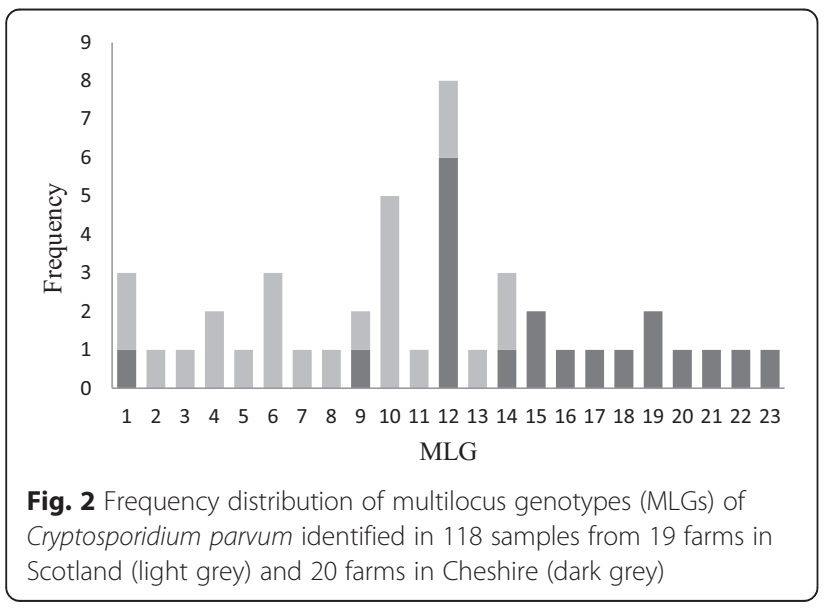

etc.) and humans; previous studies have used our trialled markers to successfully type both human and bovinederived C. parvum [12].

Three additional markers, MSA, MSD and MSF, were applied to representatives of the 23 MLGs identified in the current study using primers reported in the literature [11]; all 3 of these additional markers were monoallelic in our samples producing fragments of $229 \mathrm{bp}$, $274 \mathrm{bp}$ and $156 \mathrm{bp}$ respectively (data not shown). These sizes correspond with reported allele sizes for these markers [11]. In the current study only isolates of gp60 subtype IIa were tested; cattle in other countries including Portugal $[29,30]$ have been shown to infrequently shed gp60 allele IId, although this allele has not been reported to date in UK cattle. Human C. parvum is most commonly gp60 allele IIa in the UK [6] but other alleles are also occasionally identified, notably IId and, rarely, IIc [6]. More work would be required to determine the performance of the markers proposed in the current study in non-IIa gp60 subtypes of C. parvum.

Calves from both Cheshire 2004 and NE Scotland 2011 cross-sectional studies appeared to shed the same predominant alleles of the markers used and allele frequency distributions were very similar, suggesting that these alleles are fairly stable in UK calf populations. It is also clear that the same alleles are mainly present in other Scottish studies of calves [12], as demonstrated in Table 5; a few additional alleles were detected, probably due to differences in study design, but in very low numbers. We also assessed available literature where the same markers and primers were used. As previously noted, this was limited by the lack of a coordinated approach to marker selection. In addition allele sizes are not always reported; where they are given, it is not possible to prove definitively that reported alleles are the same due to the previously stated problems with fragment sizing. Authors rarely state if reported sizes are sequence sizes or binned fragment sizes. A study of C. parvum in Italian livestock used the same marker combination as the current study, but there were some small variations in (second round) primer sequences (TP14 reverse and MM19 forward) [26]. These primer sequences were also applied to C. parvum samples collected from calves in Ireland 2003-2005 [22]. Interestingly in both of these studies, size and frequency of alleles reported for MM5, MM18, TP14 and MS1 were similar to those found in the current study: $94-98 \%$ was 233/260 bp for MM5, 65-95 \% was 290/296 bp for MM18, 89-95 \% was $300 / 309$ bp for TP14 and 66-99 \% was 362 bp for MS1. The amended MM19 forward primer sequence used by Drumo et al. and De Waele et al. aligns with the published reference genome for $C$. parvum and may be superior to that used in the current study. These differences also account for the small number 
of base differences detected when we aligned our allele sequences with other microsatellite sequences using BLAST.

As well as the improved typeability of MLFT over MLST as reported by Diaz et al. [13], we found MLFT also compares favourably to sequencing in terms of time and cost. Although the use of fluorescently-labelled primers adds to the cost of standard PCR, fragment analysis was economical compared to sequencing at approximately £0.80 (1 EURO, \$1.28) per read; this could have been reduced by multiplexing PCR products into one well for sizing, either using different fluorescent labels or ensuring that expected fragment sizes were sufficiently different to allow differentiation. In addition, 10/118 samples had more than one allele at one, or more, markers, which would not have been detected using direct sequence typing. This is similar to the $11.6 \%$ of infections found to be mixed in an Italian study of humans and livestock [26]. In other studies, criteria for assignment of mixed genotypes are unclear, or more stringent. For example one Scottish study defined a sample as mixed if the height of the secondary peak was $>10 \%$ of the main peak, possibly explaining why they detected a relatively high prevalence of mixed infections - up to $37 \%$ in Aberdeenshire [12]. The prevalence of mixed infections may also increase with age of animal sampled, as older animals will have been exposed to more sources of oocysts. However the advantages of MLST are that it provides greater discrimination than MLFT and both accuracy and reproducibility are superior, in single genotype infections.

Our preferred software was STRand, as we found that PeakScanner had problems with bleed-through in the event of the product being too strong. This problem can be easily detected and manually corrected using STRand. When comparing results we found minimal variation when the same sample was sized with the two different softwares. Both of these softwares are free to download.

\section{Conclusions}

We found MLFT using markers MM5, MM18, MM19 and TP14 performed well in typing bovine C. parvum, in combination with results for sequence analysis of the GP60 gene. It appears that the common alleles in cattle in the UK have been identified, although it is expected that further studies will produce new alleles which should be verified and added to the reference collection.

These markers have also shown to have good typeability and discrimination in human C. parvum (personal communication, Chalmers), and have also been used successfully in a water catchment level investigation of contamination of a water supply by Cryptosporidium oocysts [31]. Therefore we believe this tool has potential value in source attribution in the event of an outbreak of C. parvum in humans. The adoption of standardised primers is to be encouraged, and collaboration particularly at regional and national levels between public health bodies and veterinary researchers is vital. A system for standardising the sizing should be considered, potentially including a centrally-curated panel of all known alleles that can be used to calibrate machines or to be run in parallel with test samples. A standardised nomenclature system perhaps based on the number of repeats may also be helpful [32]. It is likely that advancing technology such as next generation sequencing may supersede this tool; however this is not currently economically or practically feasible, particularly in the timeframe required in the event of an outbreak. The process of developing any integrated scheme will lay the foundations for future collaboration, as long as participants maintain a flexible and open-minded approach to future technological developments.

\section{Competing interests}

The authors declare that they have no competing interests.

\section{Authors' contributions}

$\mathrm{EH}$ conceived and coordinated the study, carried out some of the laboratory work, analysed the results and drafted the manuscript. JG contributed to the planning of the study and carried out most of the laboratory work. MB and LM contributed to the planning of the study, carried out some of the laboratory work and edited the manuscript. RC, NJ, LI and FK contributed to the planning of the study and edited the manuscript. All authors approved the final manuscript.

\section{Acknowledgements}

This project was funded by the Scottish Government (SPASE WS3.2.3). The field study in Cheshire was funded as part of a BBSRC Studentship at the University of Liverpool (EH) and the field study in Northeast Scotland was part-funded by Quality Meat Scotland. RC and MB were funded by the Department for Environment, Food and Rural Affairs (DEFRA) and by the Higher Education Funding Council for England (HEFCE) under grant VTRI VT0103. We are indebted to the farmers and veterinary surgeons who kindly provided material. LM is a Royal Society University Research Fellow (UF090083). Rob Christley is supported by the National Institute for Health Research Health Protection Research Unit in Emerging and Zoonotic Infections.

\section{Author details}

${ }^{1}$ Moredun Research Institute, Pentlands Science Park, Bush Loan, Penicuik, Edinburgh EH26 OPZ, UK. ${ }^{2}$ School of Veterinary Medicine and Science, University of Nottingham, Sutton Bonington Campus, Loughborough LE12 5RD, UK. ${ }^{3}$ Institute of Infection and Global Health, University of Liverpool, NIHR Health Protection Research Unit in Emerging and Zoonotic Infections, Leahurst Campus CH64 7TE, Liverpool L69 7BE, UK. ${ }^{4}$ Roslin Institute, Royal (Dick) School of Veterinary Studies, University of Edinburgh, Easter Bush, Midlothian EH25 9RG, UK. Institute of Biodiversity, Animal Health and Comparative Medicine, University of Glasgow, Bearsden Road, Glasgow G61 $1 \mathrm{QH}$, UK.

Received: 10 December 2014 Accepted: 22 September 2015 Published online: 01 October 2015

\section{References}

1. Chalmers RM, Katzer F. Looking for Cryptosporidium: the application of advances in detection and diagnosis. Trends Parasitol. 2013;29:237-51.

2. Garber LP, Salman MD, Hurd HS, Keefe T, Schlater JL. Potential risk factors for Cryptosporidium infection in dairy calves. J Am Vet Med Assoc. 1994;205:86-91.

3. Quilez J, Sanchez-Acedo C, del Cacho E, Clavel A, Causape AC. Prevalence of Cryptosporidium and Giardia infections in cattle in Aragon (northeastern Spain). Vet Parasitol. 1996;66:139-46. 
4. Strong WB, Gut J, Nelson RG. Cloning and sequence analysis of a highly polymorphic Cryptosporidium parvum gene encoding a 60-kilodalton glycoprotein and characterization of its 15 - and 45 -kilodalton zoite surface antigen products. Infect Immun. 2000;68:4117-34.

5. Sulaiman IM, Hira PR, Zhou L, Al-Ali FM, Al-Shelahi FA, Shweiki HM, et al. Unique endemicity of cryptosporidiosis in children in Kuwait. J Clin Microbiol. 2005;43:2805-9.

6. Chalmers RM, Smith RP, Hadfield SJ, Elwin K, Giles M. Zoonotic linkage and variation in Cryptosporidium parvum from patients in the United Kingdom. Parasitol Res. 2011;108:1321-5.

7. Brook EJ, Hart CA, French NP, Christley RM. Molecular epidemiology of Cryptosporidium subtypes in cattle in England. Vet J. 2009;179:378-82.

8. Gatei W, Hart CA, Gilman RH, Das P, Cama V, Xiao L. Development of a multilocus sequence typing tool for Cryptosporidium hominis. J Eukaryot Microbiol. 2006;53 Suppl 1:S43-8.

9. Gatei W, Das P, Dutta P, Sen A, Cama V, Lal AA, et al. Multilocus sequence typing and genetic structure of Cryptosporidium hominis from children in Kolkata, India. Infect Genet Evol. 2007;7:197-205.

10. Gatei W, Barrett D, Lindo JF, Eldemire-Shearer D, Cama V, Xiao L. Unique Cryptosporidium population in HIV-infected persons, Jamaica. Emerg Infect Dis. 2008:14:841-3.

11. Tanriverdi S, Markovics A, Arslan MO, Itik A, Shkap V, Widmer G. Emergence of distinct genotypes of Cryptosporidium parvum in structured host populations. Appl Environ Microbiol. 2006;72:2507-13.

12. Morrison LJ, Mallon ME, Smith HV, MacLeod A, Xiao L, Tait A. The population structure of the Cryptosporidium parvum population in Scotland: a complex picture. Infect Genet Evol. 2008:8:121-9.

13. Diaz P, Hadfield SJ, Quilez J, Soilan M, Lopez C, Panadero R, et al. Assessment of three methods for multilocus fragment typing of Cryptosporidium parvum from domestic ruminants in north west Spain. Vet Parasitol. 2012;186:188-95.

14. Pasqualotto AC, Denning DW, Anderson MJ. A cautionary tale: Lack of consistency in allele sizes between two laboratories for a published multilocus microsatellite typing system. J Clin Microbiol. 2007;45:522-8.

15. Robinson G, Chalmers RM. Assessment of polymorphic genetic markers for multi-locus typing of Cryptosporidium parvum and Cryptosporidium hominis. Exp Parasitol. 2012;132:200-15.

16. Brook E, Hart CA, French N, Christley R. Prevalence and risk factors for Cryptosporidium spp. infection in young calves. Vet Parasitol. 2008;152:46-52.

17. Xiao L, Escalante L, Yang C, Sulaiman I, Escalante AA, Montali RJ, et al. Phylogenetic analysis of Cryptosporidium parasites based on the small-subunit rRNA gene locus. Appl Environ Microbiol. 1999;65:1578-83.

18. Brennan ML: Contacts between cattle farms and their role in pathogen transmission. PhD Thesis. University of Liverpool; 2008. http://ethos.bl.uk OrderDetails.do?uin=uk.bl.ethos.501696.

19. Mallon M, MacLeod A, Wastling J, Smith H, Reilly B, Tait A. Population structures and the role of genetic exchange in the zoonotic pathogen Cryptosporidium parvum. J Mol Evol. 2003;56:407-17.

20. Mallon ME, MacLeod A, Wastling JM, Smith H, Tait A. Multilocus genotyping of Cryptosporidium parvum Type 2: population genetics and sub-structuring. Infect Genet Evol. 2003;3:207-18.

21. Hunter PR, Gaston MA. Numerical index of the discriminatory ability of typing systems: an application of Simpson's index of diversity. J Clin Microbiol. 1988;26:2465-6.

22. De Waele V, Van den Broeck F, Huyse T, McGrath G, Higgins I, Speybroeck $\mathrm{N}$, et al. Panmictic structure of the Cryptosporidium parvum population in irish calves: influence of prevalence and host movement. Appl Environ Microbiol. 2013;79:2534-41.

23. Quilez J, Vergara-Castiblanco C, Monteagudo L, Del Cacho E, Sanchez-Acedo C. Multilocus fragment typing and genetic structure of Cryptosporidium parvum Isolates from diarrheic preweaned calves in Spain. Appl Environ Microbiol. 2011;77:7779-86.

24. Struelens MJ. Consensus guidelines for appropriate use and evaluation of microbial epidemiologic typing systems. Clin Microbiol Infect. 1996;2:2-11.

25. van Belkum A, Tassios PT, Dijkshoorn L, Haeggman S, Cookson B, Fry NK, et al. Guidelines for the validation and application of typing methods for use in bacterial epidemiology. Clin Microbiol Infect. 2007;13 Suppl 3:1-46.

26. Drumo R, Widmer G, Morrison LJ, Tait A, Grelloni V, D'Avino N, et al. Evidence of Host-Associated Populations of Cryptosporidium parvum in Italy. Appl Environ Microbiol. 2012;78:3523-9.
27. Widmer G, Sullivan S. Genomics and population biology of Cryptosporidium species. Parasite Immunol. 2012;34:61-71.

28. Santin M, Trout JM, Xiao L, Zhou L, Greiner E, Fayer R. Prevalence and age-related variation of Cryptosporidium species and genotypes in dairy calves. Vet Parasitol. 2004;122:103-17.

29. Alves M, Xiao L, Sulaiman I, Lal AA, Matos O, Antunes F. Subgenotype analysis of Cryptosporidium isolates from humans, cattle, and zoo ruminants in Portugal. J Clin Microbiol. 2003;41:2744-7.

30. Alves M, Xiao L, Antunes F, Matos O. Distribution of Cryptosporidium subtypes in humans and domestic and wild ruminants in Portugal. Parasitol Res. 2006;99:287-92.

31. Wells B, Shaw H, Hotchkiss E, Gilray J, Ayton R, Green J, et al. Prevalence, species identification and genotyping Cryptosporidium from livestock and deer in a catchment in the Cairngorms with a history of a contaminated public water supply. Parasit Vectors. 2015:8:66.

32. Nadon CA, Trees E, Ng LK, Moller NE, Reimer A, Maxwell N, et al. Development and application of MLVA methods as a tool for inter-laboratory surveillance. Euro Surveill. 2013;18:20565.

33. Khramtsov NV, Tilley M, Blunt DS, Montelone BA, Upton SJ. Cloning and analysis of a Cryptosporidium parvum gene encoding a protein with homology to cytoplasmic form Hsp70. J Eukaryot Microbiol. 1995:42:416-22.

\section{Submit your next manuscript to BioMed Central and take full advantage of:}

- Convenient online submission

- Thorough peer review

- No space constraints or color figure charges

- Immediate publication on acceptance

- Inclusion in PubMed, CAS, Scopus and Google Scholar

- Research which is freely available for redistribution 